\title{
Is the SNAP-II score useful for predicting mortality in mechanically ventilated in neonates within the first 12 hours of admission?
}

\author{
Narongsak Nakwan ${ }^{\mathrm{a}, \mathrm{b}}$, Jeerawan Wannaro ${ }^{\mathrm{b}}$, Pornpreenun Chaiwiriyawong ${ }^{\mathrm{b}}$ \\ ${ }^{a}$ Neonatal Intensive Care Unit, Department of Pediatrics, Hat Yai Medical Education Center, Hat Yai \\ Hospital, Songkhla 90110, Thailand \\ ${ }^{b}$ Department of Pediatrics, Hat Yai Medical Education Center, Hat Yai Hospital, Songkhla 90110, \\ Thailand
}

\begin{abstract}
Background: Respiratory failure is a most serious neonatal disorder. A severity of illness assessment should be performed for mechanically ventilated infants to predict the probability of hospital mortality and to assist clinical decision making.

Objectives: To evaluate the ability of the Score for Neonatal Acute Physiology, version II (SNAP-II) to predict the mortality risk for the infants who are mechanically ventilated with positive pressure ventilation (PPV) at a neonatal intensive care unit (NICU).

Methods: A prospective observational cohort study was performed in a 12-bed level III NICU at Hat Yai Hospital between January 2010 and December 2010.

Results: During the study period, 46 infants were ventilated with PPV at admission. Of these, 8 died (17\%) and 30 survived (83\%). The overall mean SNAP-II score was significantly higher in the group of infants who died compared with those who survived ( $36.5 \pm 11.5$ vs. $22.3 \pm 13.6, P<0.01$, respectively). The area under the receiver operating characteristic curve for the SNAP-II was 0.79 (95\% confidence interval (CI) 0.66 to $0.92, P=0.01$ ). Every one-point increment of SNAP-II was associated with an odds ratio of 1.08 (95\% CI 1.02 to $1.14, P<0.01$ ). The stratification of the two sets of scores when compared between groups showed that infants who had SNAP-II scores of $\geq 32$ had the highest mortality risk with an odds ratio of 9.7 (95\% CI 1.7 to 56.5) with sensitivity of $75.0 \%$ and specificity of $76.3 \%$.
\end{abstract}

Conclusion: SNAP-II scores can significantly predict mortality in infants requiring mechanical PPV.

Keywords: Neonatal respiratory failure, neonatal mortality, mechanical ventilation, severity of illness index, SNAP-II

Respiratory disorder is the most common morbidity and cause of mortality in the neonatal population [1-2]. Mechanical positive pressure ventilation is the most useful treatment for neonatal respiratory failure. Given the improvements in neonatal care in neonatal intensive care units (NICUs), it is imperative that there be strict quality control to identify the groups at the greatest risk of death and to ensure the adequacy of treatment and the planning and rational use of resources. Differences in mortality rates within NICUs can be explained by the severity of illness of patients treated when suffering from

Correspondence to: Narongsak Nakwan, Neonatal Intensive Care Unit, Department of Pediatrics, Hat Yai Medical Education Center, Hat Yai Hospital, Songkhla 90110, Thailand.

E-mail:nnakwan@hatyaihospital.go.th various conditions [2]. The use of prognostic indicators is an essential quality criterion in the care of critically ill patients. Although birth weight, gestational age, and Apgar scores are the traditional general assessment tools for newborns [3], the relationship between these parameters and mortality is insufficiently precise for usefully reliable assessment of mortality risk. The Score for Neonatal Acute Physiology, version II (SNAP-II) was recently simplified and revalidated for use in predicting hospital mortality in infants who were admitted to NICUs by assessing 6 physiological variables within the first 12 hours following admission $[4,5]$. The objective of this study was to determine the performance of the SNAP-II in predicting mortality in infants requiring mechanical ventilation at NICU admission. 


\section{Patients and methods}

This prospective observational cohort study was performed in the 12-bed level III NICU at Hat Yai Hospital between January 2010 and December 2010. Infants who were intubated before NICU admission were included in the study. Infants with an additional major congenital anomaly or cyanotic heart disease, or who died within 12 hours following NICU admission, were excluded. The study including prospectively abstracting of patient information from clinical charts was approved by the Hat Yai Hospital Ethics Committee.

The basic characteristics of the patients were noted, and the worst readings of six physiological parameters (lowest blood pressure, lowest temperature, lowest $\mathrm{PaO}_{2} / \mathrm{FiO}_{2}$ ratio, lowest serum $\mathrm{pH}$, urine output, and seizures) in the first 12 hours following NICU admission for SNAP-II were abstracted from clinical and laboratory records, using the variable definitions reported in the original SNAP-II report [4].

\section{Statistical analysis}

Data were evaluated using descriptive statistics (mean and standard deviation, median and range (minimum-maximum), or frequency and percentage, as appropriate). Student's $t$ test was used for analysis of continuous variables. Differences in proportions between groups were determined using a chi-square or Fisher's exact test. We conducted multivariate logistic regression analyses to test whether variables were independently associated with mortality.

Performance of the SNAP-II score was evaluated by assessing discrimination and calibration. Discrimination was tested by the area under the receiver operating characteristic (ROC) curve (AUC), which was also used to identify the best cut-off points of SNAP-II scores [6]. Acceptable discrimination is represented by an AUC of 0.75 or more [7]. For calibration, the goodness-of-fit of the model was examined by the Hosmer-Lemeshow test [8]. This method is useful in assessing deviations between the observed and expected numbers of deaths. Interpretation of this test shows that if the difference between the observed and expected mortality is not significant $(P>0.05)$, then they are comparable. This means that the model has a significantly good calibration if $P>0.05$. Cox proportional hazard regression analysis was performed to assess the effect of SNAP-II score and oxygen index (OI) point to mortality. Hazard ratios (HR) with 95\% confidence intervals (CI) were used to quantify the associations. Data analyses were performed using SPSS version 13.0 (SPSS Inc, Chicago, IL).

\section{Results}

During the study period, 626 infants were admitted to the NICU, of whom 241 required mechanical positive pressure ventilation. Of these 241 infants, 46 (19.1\%) were initially intubated before NICU admission. Table 1 presents the basic characteristics of the study population. The mean gestational age and birth weight were $35.4 \pm 4.5$ weeks and 2,401 $\pm 1,022$ g, respectively, and 33 infants (72\%) were male. Eight (18\%) were referred to our institution. Respiratory distress syndrome (RDS) was the most common etiology of respiratory disorder in the ventilated infants in our study (19 of 46, 41\%), and this group had a mortality rate of $13 \%$. The second most common cause of respiratory disorder in our study was meconium aspiration syndrome ( 13 of $46,28 \%$ ), partly complicated by persistent pulmonary hypertension of the newborn (PPHN) (3 of 13, 23\%). In univariate analysis, comparing with infants who survived, the infants who died had lower OI, lower SNAP-II, lower birth weight, less length of stay. In multivariate analysis, there was no detectable factor found associated with mortality.

The study population had a mean SNAP-II of $22.8 \pm 14.2$. The mean SNAP-II was significantly higher in nonsurvivors than in survivors $(36.5 \pm 11.5$ vs. $22.3 \pm 13.6, P<0.01$, respectively). Infants classified as having 'severe illness' (a SNAP-II of $\geq 32$ ) had the greatest mortality risk with HR of 9.7 (95\% confidence interval (CI) 1.7 to 56.5). Every one point increase in the SNAP score increased the HR of mortality by 1.08 (95\% CI 1.02 to 1.14 , $P<0.01)$. On the other hand, the median oxygen index (OI) score was significantly higher in nonsurvivors than in survivors (25.3 (range: 11.6-124.2) vs. 6.0 (range: 1.8-40.8), $P<0.01$, respectively). Every one point increase in the OI increased the HR of mortality by 1.04 (95\% CI 1.02 to $1.06, P<0.01$ ).

The AUC of ROC for the SNAP-II, and OI was 0.79 (95\% CI 0.66 to $0.92, P=0.01)$, and 0.85 (95\% CI 0.76 to $0.98, P=0.01$ ), respectively. The goodnessof-fit by the Hosmer-Lemeshow test showed that the SNAP-II test and OI were well calibrated in our study to assess deviation between the observed and expected numbers of death cases $(P=0.34$, and $P=0.22$, respectively). A SNAP-II cut-off of 32 was 
Table 1 Characteristics of the 46 study infants

\begin{tabular}{llll}
\hline Characteristic & Survivors (n= 38) & Non-survivors (n= 8) & $P$ \\
\hline Gestational age (weeks), mean \pm SD & $35.0 \pm 4.5$ & $37.4 \pm 4.4$ & 0.20 \\
Birth weight (g), mean \pm SD & $2,310 \pm 1042$ & $2,838 \pm 852$ & 0.09 \\
$\quad \leq 999$ g & $1(3 \%)$ & $1(13 \%)$ & 0.32 \\
$\quad 1,000-1,499 g$ & $12(37 \%)$ & 0 & 0.09 \\
$\quad \geq 1,500$ g & $25(66 \%)$ & $7(88 \%)$ & 0.40 \\
Male, n (\%) & $27(71 \%)$ & $6(75 \%)$ & 1.00 \\
Outborn, n (\%) & $6(16 \%)$ & $2(25 \%)$ & 0.61 \\
Apgar Score at 1 min, mean \pm SD & $7 \pm 2$ & $7 \pm 3$ & 0.46 \\
Apgar Score at 5 min, mean \pm SD & $8 \pm 1$ & $7 \pm 3$ & 0.71 \\
Initial temperature ( ${ }^{\circ}$ C), mean \pm SD & $36.5 \pm 0.8$ & $36.8 \pm 0.7$ & 0.18 \\
Small for gestational age, n (\%) & $3(8 \%)$ & $1.0(13 \%)$ & 1.00 \\
Oxygen Index, median (range) & $6.0(1.8-40.8)$ & $25.3(11.6-124.2)$ & $<\mathbf{0 . 0 1}$ \\
Causes of respiratory disorder, n (\%) & & & \\
$\quad$ Respiratory distress syndrome & $18(47 \%)$ & $1(13 \%)$ & 0.12 \\
$\quad$ Meconium aspiration syndrome & $10(26 \%)$ & $3(38 \%)$ & 0.67 \\
$\quad$ Congenital pneumonia & $7(18 \%)$ & $2(25 \%)$ & 1.00 \\
$\quad$ Transient tachypnea of the newborn & $2(5 \%)$ & $2(25 \%)$ & 0.13 \\
$\quad$ Subgaleal/hemorrhage with shock & $1(3 \%)$ & 0 & 1.00 \\
Length of stay, median (days) & $30.0(8.3-99.6)$ & $1.9(1.0-20.4)$ & $<\mathbf{0 . 0 1}$ \\
\hline
\end{tabular}

derived from the ROC analysis for prediction of mortality. The sensitivity, specificity, and positive and negative predictive values (NPV) of SNAP-II $\geq 32$ in predicting mortality were $75.0 \%, 76.3 \%, 40.0 \%$, and $93.5 \%$, respectively.

\section{Discussion}

Currently, the treatment modalities of NICUs for infants with respiratory failure may influence mortality. Hence, early identification of infants with respiratory disorders that present a high mortality risk may be helpful in guiding clinical decision-making and resource allocation. In the present study, the SNAP-II was studied to determine its usefulness in predicting mortality in mechanically ventilated infants in our NICU. We found that the SNAP-II offered an acceptable discriminative power with an AUC of 0.79, and had a significantly good calibration for both expected and observed mortalities.

An association between mortality and higher SNAP-II in sick infants has been previously reported and the authors of the current study have previously reported the SNAP-II accurately predicted mortality in infants with PPHN $[2,9]$. Those results were similar to those in the present study, because in both studies the discrimination levels were acceptable (AUC 0.72 vs. 0.79, respectively). Our results were consistent with those in the study by Skarsgard et al. who showed the discrimination ability of SNAP-II to predict mortality in congenital diaphragmatic hernia had an AUC of 0.76 [10]. Ma et al. [2] reported the epidemiology of respiratory distress associated the severity illness in late preterm or term infants. Their study had a similar study population to ours, and found that a higher SNAP-II was significantly correlated with death, with an odds ratio (OR) from multivariate logistic regression analysis of 1.07 (95\% CI 1.04 to 1.10), nearly the same as the HR from the present study of 1.08 (95\% CI 1.02 to 1.14). Unfortunately, Ma et al. did not analyze the discriminative ability of the SNAP-II. In the present study, the evaluation of the SNAP-II with a cut-off value of 32 in the ROC had acceptable levels of sensitivity and specificity as a predictor of mortality in mechanically ventilated infants. This cut-off point was less than the cut-off point in infants with PPHN that we found, probably because the infants with PPHN were more severely ill and had a higher mortality rate [9].

The results of our study confirm that the SNAPII and OI can be useful predictors of mortality in infants with respiratory failure. Our results also confirm that OI can be as useful as SNAP-II in predicting mortality in sick infants [2, 11]. Therefore, we suggest using SNAP-II and OI together in assessing infants with respiratory failure within 12 hours of admission, because both are relatively simple 
and inexpensive, and results can be obtained within only a few minutes. In addition, birth weight was a also factor associated with mortality. However, the multivariable analysis was inconclusive, probably because of the small sample size. Morbidity prediction with the SNAP-II has been previously examined in subgroups of illnesses such as chronic lung disease or intraventricular hemorrhage [12].

Our study has some limitations. The most significant is that our mortality data was based on short term outcomes only, i.e., death or hospital discharge. Ultimately, tools used for outcome prediction should reflect longer-term outcomes of neonatal respiratory failure including growth and potential neurodevelopmental effects of the illness being assessed. Moreover, the sample size in this study was small. Although the SNAP-II has been shown to be a useful predictor of mortality outcome for a number of neonatal respiratory diseases, a larger sample size is required to validate the SNAP-II's ability to predict mortality in infants with respiratory failure.

In conclusion, the SNAP-II has good discriminatory power in predicting mortality in infants with respiratory failure who need mechanical positive pressure ventilation after delivery. The SNAP-II score can be applied with an adequate degree of accuracy for severity assessment in clinical practice.

\section{Acknowledgments}

The authors thank Dr. Chulalak Komonti, Department of Developmental Research, Siriraj Hospital Faculty of Medicine, Mahidol University, Bangkok, Thailand, and Mr. David Patterson for help with English editing of this manuscript.

No authors have any conflict of interest.

\section{References}

1. Qian L, Liu C, Zhuang W, Guo Y, Yu J, Chen H, et al. Neonatal respiratory failure: a 12-month clinical epidemiology study from 2004 to 2005 in China. Pediatrics. 2008; 121:e1115-24.

2. Ma X-L, Xu X-F, Chen C, Yan C-Y, Liu Y-M, Xiong H, et al. Epidemiology of respiratory distress and the illness severity in late preterm or term infants: a prospective multi-center study. Chin Med J. 2010; 123:2776-80.

3. Casey BM, McIntire DD, Leveno KJ. The continuing value of the Apgar Score for the assessment of newborn infants. N Engl J Med. 2001; 344:467-71.

4. Richardson DK, Corcoran JD, Escobar GJ, Lee SK. SNAP-II and SNAPPE-II: Simplified newborn illness severity and mortality risk scores. J Pediatr. 2001; 138: 92-100.

5. Zupancic JA, Richardson DK, Horbar JD, Carpenter JH, Lee SK, Escobar GJ, et al. Revalidation of the Score for Neonatal Acute Physiology in the Vermont Oxford Network. Pediatrics. 2007; 119:e156-63.

6. Hanlay JA, McNeil BJ. The meaning and use of the area under the receiver operating characteristic (ROC) curve. Radiology. 1982; 143:29-36.

7. Zweig $\mathrm{MH}$, Campbell G. Receiver-operating characteristic (ROC) plots: a fundamental evaluation tool in clinical medicine. Clin Chem. 1993; 39:561-77.

8. Hosmer DW, Lemeshow S. Applied logistic regression. Wiley, New York, 1989.

9. Nakwan N, Nakwan N, Wannaro J. Predicting mortality outcome in infants with persistent pulmonary hypertension of the newborn with the Score for Neonatal Acute Physiology-Version II (SNAP-II) in Thai neonates. J Perinat Med. 2011; 39:311-35.

10. Skarsgard ED, MacNab YC, Qiu Z, Little R, Lee SK, The Canadian Neonatal Network. SNAP-II predicts mortality among infants with congenital diaphragmatic hernia. J Perinatol. 2005; 25:315-9.

11. Kumar D, Super DM, Fajardo RA, Stork EE, Moore JJ, Saker FA. Predicting outcome in neonatal hypoxic respiratory failure with the Score for Neonatal Acute Physiology (SNAP) and highest oxygen index (OI) in the first 24 hours of admission. J Perinatol. 2004; 24: 376-81.

12. Chien LY, Whyte R, Thiessen P, Walker R, Brabyn D, Lee SK, et al. SNAP-II predicts severe intraventricular hemorrhage and chronic lung disease in the Neonatal Intensive Care Unit. J Perinatol. 2002; 22:26-30. 